\title{
过渡金属催化的多炔基化合物高选择性亲电环异构化反应研究
}

\author{
朱辉严冰玉 曹杨陈知远*
}

(江西师范大学化学化工学院 功能有机小分子教育部重点实验室 南昌 330022)

\begin{abstract}
摘要 对我们研究小组近几年来在过渡金属催化的复合炔基化合物的高选择性环异构化反应方面取得的成果进行了 小结. 通过底物与亲电试剂的分子间串联亲电环化反应，有效地构建了官能团化的苯并 $[a]$ 芴醇和密集三取代的卤代炔 基菜酮环结构单元衍生物. 基于此研究基础, 又发展了一类过渡金属催化的 1,6-二炔醇酯或 1,5-二炔类化合物的分子内 环异构化反应，构建了官能团化的亚甲基狮结构单元以及含三氟甲硫基结构的狮衍生物. 此外对影响反应的可能因素 也做了讨论，提出了可能的反应机理.
\end{abstract}

关键词＼cjkstart过渡金属催化; 多炔基化合物; 环异构化; 串联反应

\section{Transition-Metal-Catalyzed Highly Selective Electrophilic Cycloisomerization Reaction of Multi-alkynyl Compounds}

\author{
Zhu, Hui Yan, Bingyu Cao, Yang Chen, Zhiyuan* \\ (Key Laboratory of Functional Small Organic Molecules, Ministry of Education, College of Chemistry \& \\ Chemical Engineering, Jiangxi Normal University, Nanchang 330022)
}

\begin{abstract}
This article summarized our recent progress in transition-metal-catalyzed highly selective cycloisomerization reactions of 1,5- and/or 1,6-diylol compounds, which selectively provided useful access to polyfunctionalized benzo[a]fluorenol and densely trisubstituted naphthalene derivatives. Based on these results, we have also developed transition-metal-catalyzed tandem cycloisomerization reactions of 1,6-diynl esters or 1,5-diyls, affording a series of methelene benzofulvenes derivatives and trifluoromethanesulfanyl benzofulvenes in chemo- and regio-selective manner. In addition, the factors that may affect the reaction processes were studied, and the possible reaction mechanisms are proposed.
\end{abstract}

Keywords transition-metal-catalyzed; multi-alkynyl compounds; cycloisomerization; tandem reaction

多炔基化合物具有丰富的碳链不饱和度，因此利用 多炔基化合物可以高选择性地构建环状化合物 ${ }^{[1]}$. 含有 炔基的化合物的环化反应通常操作简单、安全，反应条 件较温和, 反应产物的官能团和结构多样性也可以很容 易引入. 基于以上优点, 通过利用多炔基化合物为原料 合成环状化合物的反应很早引起了有机合成化学家的 广泛兴趣. 串联反应是指两个或两个以上组分参与的, 经过连续化学反应一锅法引入多个基团、同时构建多个 化学键得到目标产物的新型有机反应. 这类反应不仅具 有高原子经济性, 而且具有良好的反应过程经济性. 串 联反应不需要中间体的分离与提纯, 通过 “一锅法” 即 可合成结构复杂的目标分子, 有效地解决了有机化学反
应中的结构和效率兼容性的问题 ${ }^{[2]}$. 因此, 发展串联反 应符合绿色化学发展的趋势和要求, 是当前有机化学学 科发展的一个热点领域. 近年来, 众多研究小组相继报 道了复合炔基化合物的串联环化反应 ${ }^{[3]}$.

过渡金属催化的有机环化反应具有理想的化学、区 域或立体选择性，并且由于有 Lewis 酸性的金属离子的 存在，反应底物的活性也可以有效地得到增强. 过渡金 属如银 $(\mathrm{Ag})$ 和金 $(\mathrm{Au})$ 长久以来即被用作通行的货币，人 们称之为 “铸币金属” . 这几种后过渡金属的单质具有 高的氧化电势, 因而相当稳定; 但是由于外层价电子比 较容易失去，内层轨道电子的屏蔽效应以及反馈效应， 使得氧化态的金属离子空的 $\mathrm{d}$ 轨道有较强接受电子的能

*E-mail: zchen@jxnu.edu.cn

Received December 31, 2014; revised February 1, 2015; published online February 2, 2015.

Project supported by the National Natural Science Foundation of China (Nos. 21202065, 21462022), and the Natural Science Foundation of Jiangxi Province (No. 20142BAB213004).

国家自然科学基金(Nos. 21202065, 21462022)和江西省自然科学基金(No. 20142BAB213004)资助项目. 
力, 具有一定的络合配位能力. 另外, 除过渡金属元素 所特有的性质之外, 它们还有 $\mathrm{Fe}, \mathrm{Ni}, \mathrm{Cu}, \mathrm{Zn}$ 等短周期元 素类似的路易斯酸性. 因此, 氧化态的铸币金属阳离子 是相当好的电子受体, 可应用于含有多个炔基等 $\pi$ 体系 的有机催化反应. 研究表明, 含银、金、铂等元素的过 渡金属催化剂不但稳定易制备, 而且具有非常高的催化 活性, 其催化的反应具有条件较温和、底物普适性以及 较好的化学选择性 ${ }^{[4]}$. 近几年来, 基于此类过渡金属的 配合物对于各类烯、炔基团的不饱和 $\pi$ 键所呈现出的独 特的亲和性, 人们也称之为 “ $\pi$ 酸催化剂” ${ }^{[5]}$.

与此同时, 亲电试剂促进的不饱和碳链的串联环化 反应是高效构建有机碳杂环化合物的重要手段 ${ }^{[6]}$. 但是, 在传统的有机反应中, 由于亲电试剂的种类和数量相对 亲核试剂来说比较少, 并且由于单纯地使用亲电试剂启 动串联反应往往面临着化学、区域或立体选择性较差的 问题，底物的反应活性有时也成为制约反应效率的因 素, 因此基于亲电试剂促进的有机串联反应类型较为单 一. 鉴于过渡金属催化剂在有机复合炔基化合物中表现 出的良好的反应选择性以及反应催化活性, 我们设想通 过设计特定的反应体系, 在一些含有复合炔基的不饱和 $\pi$ 体系的亲电环化反应中通过加入适当的 “ $\pi$ 酸催化剂” 来调控底物的亲电型环异构化反应特性, 以此来合成具 有某些特殊结构的芳香碳杂环化合物.

在前期的研究中, 我们曾经成功地发展了一些过渡 金属催化的 ${ }^{[7]}$ 或亲电试剂促进的 ${ }^{[8]}$ 有机串联反应. 基于 这些研究基础, 最近, 我们小组又报道了在银催化剂的 作用下, 通过采用二炔醇类和三炔醇类化合物为底物与 亲电试剂 $N$-卤代丁二酰亚胺 $(\mathrm{NXS}, \mathrm{X}=\mathrm{I}, \mathrm{Br})$ 发生串联

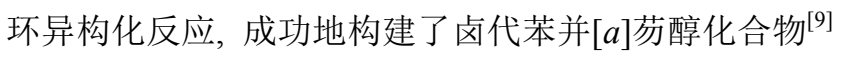
和密集多取代的萗衍生物 ${ }^{[10]}$. 此外, 我们还发现具有缺 电子属性的羰基碳原子也可作为分子内亲电试剂, 在过 渡金属铂催化剂的作用下，1,6-二炔酯类化合物可发生 分子内亲电环异构化反应, 高化学和区域选择性地构建 亚甲基苑二酮类化合物 ${ }^{[11]}$, 机理研究表明此反应的历程 涉及到一个分子内的[3,3]-重排/五元环化/超远程 1,5-酰 基迁移的历程. 最后, 我们还发现 1,5-二炔类化合物可 在三氯化铋或对甲苯磺酸的作用下，与三氟甲硫基苯胺 发生串联环化反应, 高选择性地得到了亚甲基三氟甲硫 基䒢衍生物 ${ }^{[12]}$. 上述研究结果为构建苯并 $[a$ 荡醇、菜和 亚甲基䒢衍生物等的合成提供了新的思路和方法. 本文 主要综述我们小组近几年来在该领域研究所取得的一 些成果.

\section{1 银催化的 1,6 二炔醇类化合物的亲电环化反 应合成苯并[a]芴醇化合物}

含有苯并荡骨架结构的化合物由于它们新颖的刚 性结构和大的 $\pi$ 共轭体系, 使之在材料科学和光电功能 器件方面有着非常广泛的应用. 含苯并芴骨架结构也存 在于天然产物中, 展现了良好的生物医药活性. 例如, 含有苯并 $[b]$ 芴二酮结构的 kinamycin $\mathrm{D}$ (图 1) 是一种从链 霉菌蛋白酶(streptomyces proteinase)中分离到的天然重 氮化合物，该物质对革兰氏阳性细菌生物体具有抗肿瘤 性能和抗生素活性 ${ }^{[13]}$; kinobscurinone 是一种合成醌那 霉素类药物的重要合成中间体 ${ }^{[14]}$; stealthin $\mathrm{C}$ 系列化合 物则是从 S. murayamaensis 培养中分离到的一类天然有 机分子.
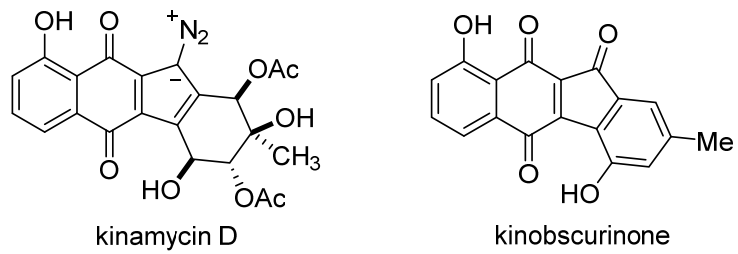

kinobscurinone
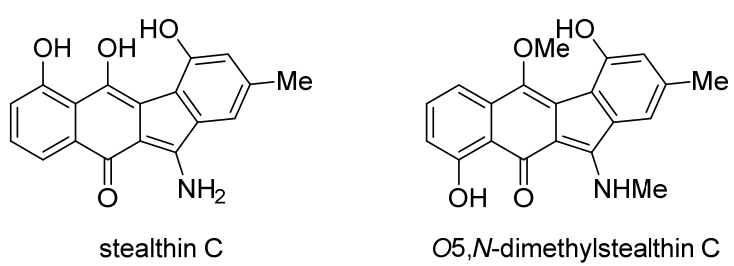

图 1 几种含苯并芴结构骨架的天然活性分子 Figure 1 Natural product-like compounds that containing benzofluorene structural motifs

基于上述醌那霉素类药物特殊的生物活性价值, 人 们对于此类苯并芴衍生物的化学合成方法也比较感兴 趣. Gould 等 ${ }^{[15]}$ 采用 $\beta$-菜甲酰胺类化合物作为合成前体, 经过一系列多步转化合成了 Kinamycin D 核心骨架. 随 后, Saá 及其合作者 ${ }^{[16]}$ 于 1999 年报道了非环状的苯并 1,6-二炔类化合物 $\mathbf{1}$ 在高温下的自由基热解反应，一步 法即可构建苯并 $[b]$ 芴分子骨架 2 (Eq. 1). 不过，由于该 反应是基于高温下的自由基重组机理，因此反应对于官 能团的兼容性非常差, 并且反应所得的构造异构体 2 无 法分离. 2009 年, 张俊良课题组 ${ }^{[17]}$ 报道了一例金、银等 “ $\pi$ 酸催化剂”催化的邻炔基苯基查尔酩的分子内串联反 应，一步法合成酰基化的苯并 $[a]$ 芴醇的反应，该反应具 有良好的转化效率, 以及中等到良好的反应产率.

我们小组 ${ }^{[9]}$ 研究发现, 在银类过渡金属催化剂的作 用下，非环状的 1,6-二炔醇类化合物 3 和 $N$-卤代丁二酰 

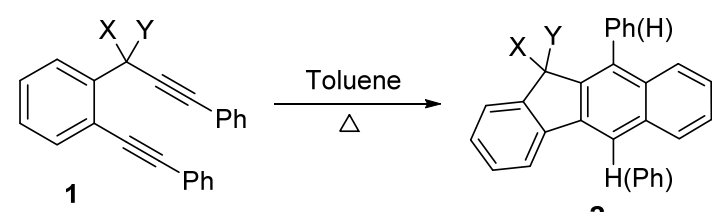

2

亚胺(NXS, X $=\mathrm{I}, \mathrm{Br})$ 发生反应, 可在十分温和的条件下 发生串联反应，高原子经济性地得到卤代苯并 $[a]$ 芴醇 类化合物 4, 并且反应具有极好的化学和区域选择性 (Eq. 2).

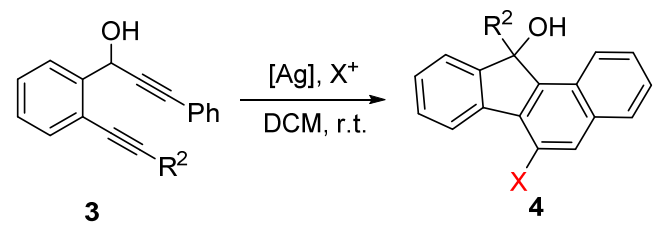

最初, 以化合物 3a 作为起始原料对各类反应参数 进行条件筛选发现, 催化剂对该反应的影响很明显(表 1). 在不加催化剂时没有反应发生(表 1, Entry 1), 这个 实验证实了过渡金属催化剂在反应转化过程中的关键 作用. 当使用各种 $\mathrm{Au}(\mathrm{I})$ 或 $\mathrm{Au}(\mathrm{III})$ 时只能比较低的收率 得到化合物 $4 \mathrm{a}$ (表 1, Entries 2 3). 令人惊奇的是, 在此 反应中银盐能给出比较高的转化效率, 当以 AgOTf (10 $\mathrm{mol} \%$ ) 作为催化剂时能以 $61 \%$ 的产率分离到目标产物 $4 \mathrm{a}$, 进一步缩小催化剂用量至 $5 \mathrm{~mol} \%$ 时反应选择性进 一步提高, 化合物 $\mathbf{4 a}$ 的收率可达 71\%(表 1, Entries 4 5). 随后, 我们对各类银催化剂进行篎选, 发现 $\mathrm{AgBF}_{4}$ 是这个反应的优化催化剂, 目标化合物 $\mathbf{4 a}$ 的产率可提 高到 $76 \%$ (表 1, Entry 8). 我们还对其他的各种参数如添 加剂、温度、溶剂以及反应液浓度等进行了篮选, 发现 反应收率都没有明显提高.

表 1 反应条件筛选及优化

Table 1 Optimization of the reaction conditions

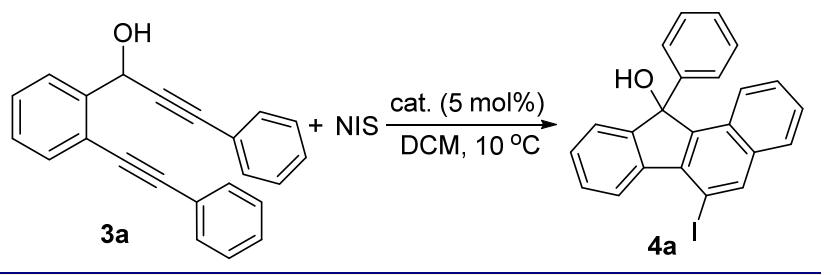

\begin{tabular}{|c|c|c|}
\hline Entry & Cat. $(x \mathrm{~mol} \%)$ & Yield/\% \\
\hline 1 & - & NR \\
\hline 2 & $\mathrm{PPh}_{3} \mathrm{Au}(\mathrm{OTf})$ & 28 \\
\hline 3 & $\mathrm{AuCl}_{3}(10)$ & $16^{\mathrm{b}}$ \\
\hline 4 & $\operatorname{AgOTf}(10)$ & 61 \\
\hline 5 & AgOTf & 71 \\
\hline 6 & $\mathrm{Ag}_{2} \mathrm{CO}_{3}$ & 29 \\
\hline 7 & $\mathrm{AgNO}_{3}$ & 31 \\
\hline 8 & $\mathrm{AgBF}_{4}$ & 76 \\
\hline 9 & $\mathrm{AgSbF}_{6}$ & 65 \\
\hline
\end{tabular}

在上述优化的条件下，我们对底物的的适应性进行 了考察(表 2). $N$-溴代丁二酰亚胺(NBS) 作为亲电试剂时 亦能顺利得到溴化的苯并 $[a]$ 荡醇. 官能团的电子效应 研究发现当 $\mathrm{R}^{1}$ 是各种吸电子基团时，反应以 $60 \% \sim 80 \%$ 产率得到化合物 $\mathbf{4 b} \sim \mathbf{4 d}$ (表 2); 不过, $\mathrm{R}^{1}$ 是给电子基团 时反应却变得很杂(表 $2,4 \mathrm{e})$. $\mathrm{R}^{3}$ 的给电子基团却对反应 影响不大, 并且此时 $N$-溴代丁二酰亚胺(NBS)作为亲电 试剂时亦能顺利得到澳化产物 $4 \mathrm{~g}$. 值得注意的是，当 $\mathrm{R}^{3}$ 是氟取代基时，反应底物的活性有所降低，相应产物 $\mathbf{4 h}$ 和 $4 \mathbf{i}$ 的产率为 $45 \% \sim 50 \%$. 反应性活泼的酰胺基团也能 在此反应中得到兼容(表 $2,4 \mathbf{j}$ ). 此外, 我们还发现当 $R^{2}$ 是给电子的芳香基团时, 产率都相对较高(表 2, 4f f). 总 体上来看, 贫电子的芳香环反应性要优于富电子的芳香 环, 我们认为造成此现象的原因可能是由于当与炔基相 连的共轭芳香环是是给电子基团时, 炔基电子云密度相 应会富集，由此导致后续的亲电环化反应步骤的反应活 性增强, 反应的区域选择性将会变差, 产率由此将会导 致有所降低.

对此反应我们给出了以下可能的串联亲电环化历 程(Scheme 1): $N$-碘代丁二酰亚胺(NIS)可在 $\mathrm{Ag}^{+}$的作用 下产生高反应活性的碘正离子 $\mathrm{I}^{+}$, 该正离子能迅速与化 合物 3a 中的炔键碳碳参键发生亲电加成反应得到碘鎓 中间体 $\mathbf{A}$, 随后, 邻位的另一个富电子的炔键会进攻此 活化的含碘正离子的 $\pi$-共轭体系, 发生分子内的六元环 化反应得到烯基正离子中间体 $\mathbf{B}^{[18]}$. 随后, 发生分子内 的傅-克型烷基环化反应得到正离子物种 $\mathbf{C}^{[19]}$. 紧接着, 此中间体物种发生去质子化反应得到中间体 D. 最后, 羟基基团被 $\mathrm{Ag}^{+}$活化离去，同时产生阳离子中间体 $\mathbf{E}$, 再经历分子内的芳香互变异构, 最终生成磑代苯并 $[a]$ 芴醇化合物 $\mathbf{4 a}$.

反应所得到的卤代苯并 $[a]$ 芴醇化合物含有可转化 性极高的碘苯结构, 因此可在钯催化剂的作用下发生偶 联反应，得到结构更趋多样性的衍生物. 例如，化合物 4a 可与对甲基苯硼酸发生 Suzuki-Miyaura 偶联反应，以 几乎定量的产率得到芳基化的苯并 $[a]$ 芴醇类衍生物 $\mathbf{5}$ (Eq. 3); 该化合物亦可发生 Sonogashira 炔基偶联反应,

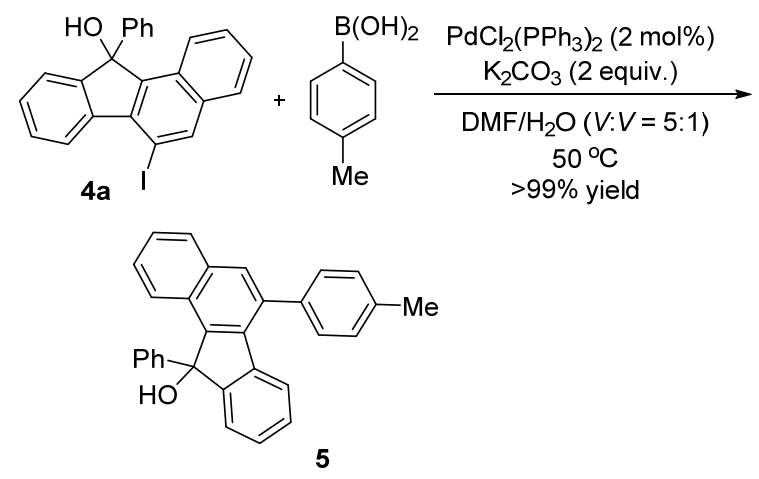


表 2 底物拓展 ${ }^{a}$

Table 2 Substrate scope

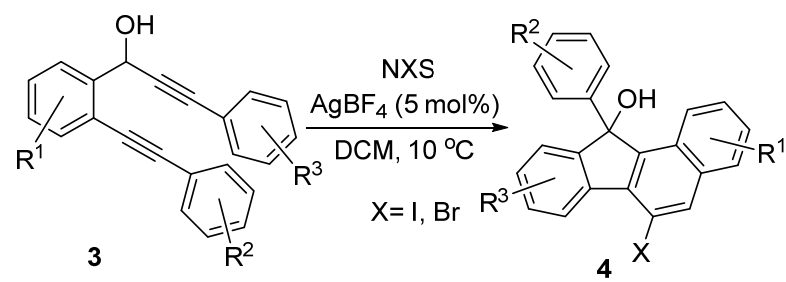

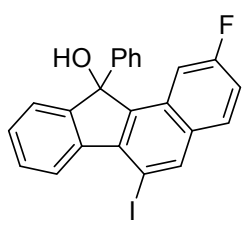

4b, $63 \%$<smiles>O=[N+]([O-])c1ccc2c3c(c(I)cc2c1)-c1ccccc1C3(O)c1ccccc1</smiles>

4d, $78 \%$<smiles>COc1ccc2c(c1)C(O)(c1ccccc1)c1c-2c(I)cc2ccccc12</smiles>

4f, $70 \%$<smiles>OC1(c2ccccc2)c2ccccc2-c2c(I)cc3ccc(F)cc3c21</smiles>

4h, $45 \%$

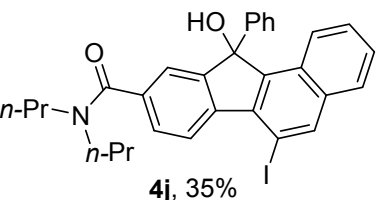

a Conditions: 3 (0.30 mmol), NXS (0.36 mol), $\mathrm{AgBF}_{4}(5 \mathrm{~mol} \%)$, $\operatorname{DCM}(2 \mathrm{~mL}), 10^{\circ} \mathrm{C}$

并以 $83 \%$ 的产率得到炔基化的苯并 $[a]$ 芴醇类衍生物 6 (Eq. 4).
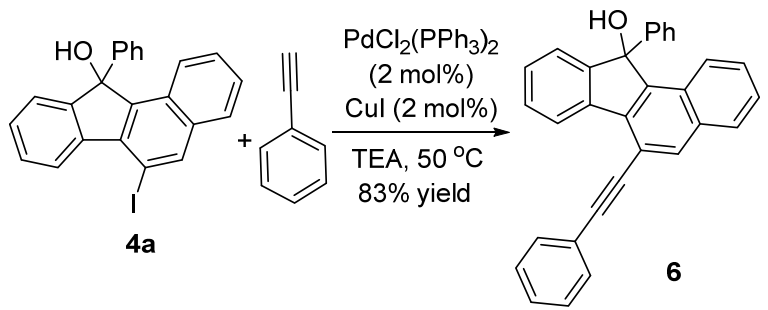

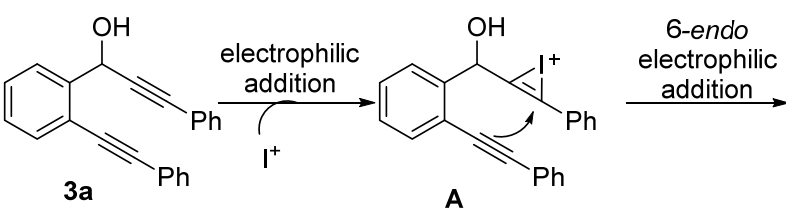

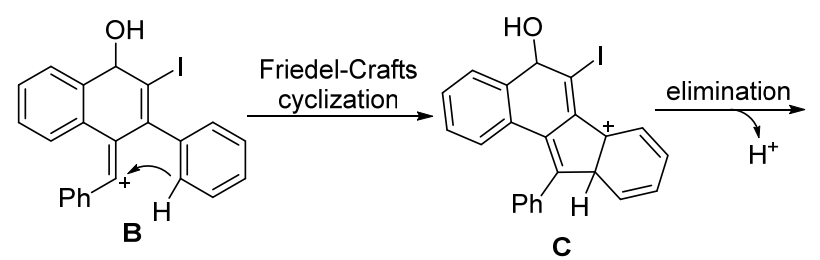

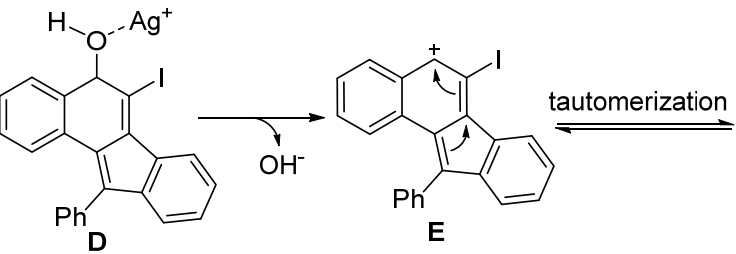<smiles>CC(O)Cc1ccc2c(c1)C(O)(c1ccccc1)c1c-2c(I)cc2ccccc12</smiles>

图式 1 串联亲电环化反应的推测机理

Scheme 1 Speculated reaction pathways of the tandem electrophilic cyclizations

\section{2 银催化的非环状三炔醇类化合物的亲电环化 反应合成密集多取代的荎衍生物}

䒺作为一种重要的稠环芳香烃化合物，在合成医 药、染料以及材料科学等方面有着重要的应用. 许多含 有荎环的化合物具有非常良好的生物或医药活性，其中 的一些含䒺化合物已经作为临床上治疗疾病的常用药 物 ${ }^{[20]}$. 例如: 䒺普生钠缓释片是一种成功上市的药物, 具有强效镇痛和抗炎作用; 荎哌地尔在治疗高血压和促 进泌尿系统结石排出方面疗效独特. 此外, 由于䒺环具 有大的 $\pi$ 电子共轭结构, 因此含荎化合物在光电功能材 料领域也具有非常广泛的应用，一些含蒜的化合物可用 于制备高性能有机/聚合物电致发光器件 ${ }^{[21]}$, 此外, 芸 在有机三阶非线性光学材料领域也有一些应用 ${ }^{[22]}$

在前述的研究中, 我们成功地发展了银催化非环化 的 1,6-二炔醇 $\mathbf{3} \mathbf{a}$ 与 $N$-卤代丁二酰亚胺(NXS)的选择性亲 电环化反应 ${ }^{[9]}$. 该反应成功的关键在于分子内的 6-endo$d i g$ 亲电碘环化反应生成关键中间体 $\mathbf{B}$ ，以及随之发生 的傅-克型亲电烷基环化反应(Scheme 2). 为了进一步 证实此反应的机制，我们设想在关键中间体 $\mathbf{B}$ 中，假如 与䒺环骨架与苯环相连的碳碳单键被其他的官能团例 如炔基碳碳三键加以延长得到中间体 $\mathbf{B}^{\prime}$ ，那么后续的 
傅-克亲电烷基环化反应将会由于碳链延长而得到抑制, 取而代之地, 碳正离子中间体将可能原位羟基化, 并随 后发生芳香异构化反应，最终将得到一类密集三取代的 卤化炔基菜酮衍生物 8 (Scheme 2). 引起我们研究兴趣 的是, 所得到的化合物 $\mathbf{8}$ 中分别含有高反应活性的邻炔 基卤代䒺和邻炔基甲基酮结构片段，此两类结构均具有 十分优异的反应活性, 可作为合成中间体进一步发生其 他的转化, 得到结构更加多样的芳香化合物 ${ }^{[10]}$.

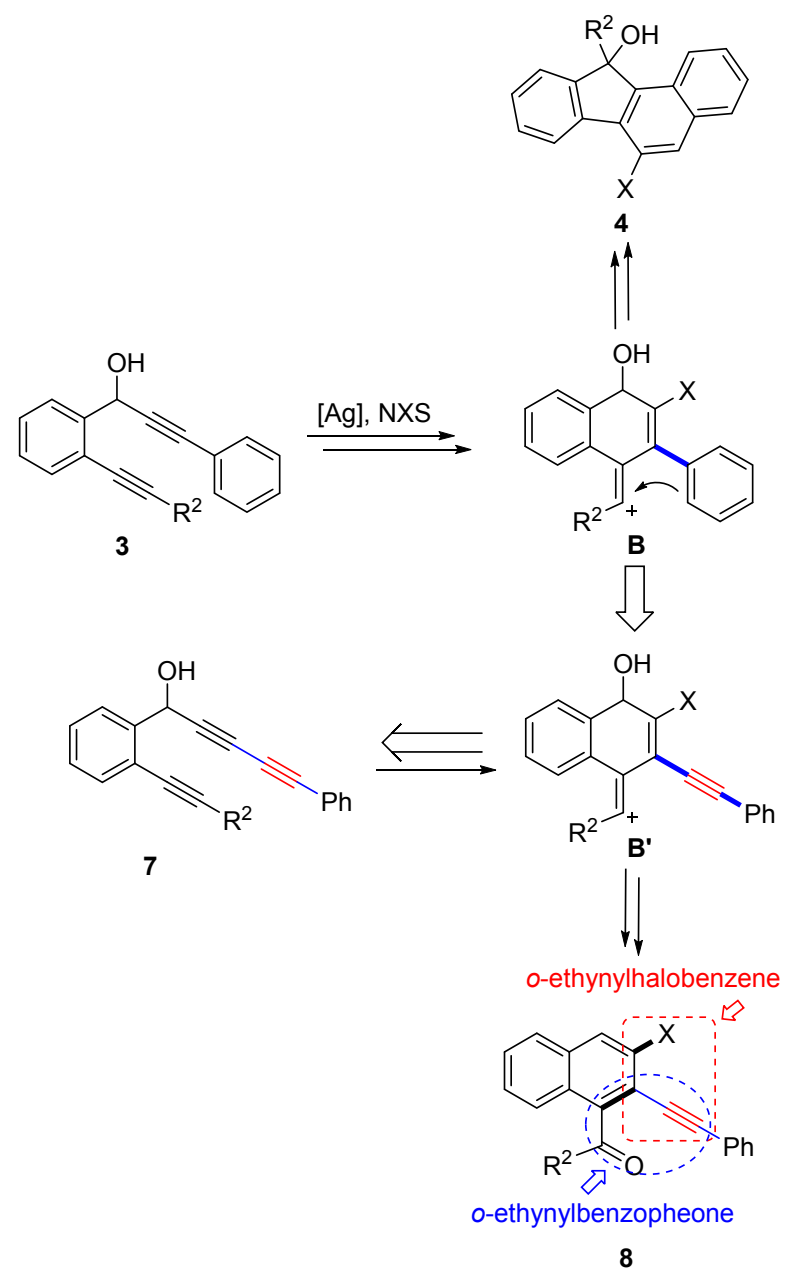

图式 2 反应设想

Scheme 2 Research design

我们设计合成了 $1,3,5$-三炔醇化合物 $7 \mathbf{a}$ 作为反应底 物, 设想通过笁选合适的反应条件来引发新型串联亲电 环化反应. 我们以前期的研究结果为基础, 发现当以 $\mathrm{AgBF}_{4}$ 为催化剂, NIS 为亲电试剂时, 反应以 $40 \%$ 的分离 收率得到了预期的目标䒺衍生物 $8 \mathbf{a}$ (表 3, Entry 1). 空白 实验表明, 当无过渡金属存在时, 反应完全不能发生 (表 3, Entry 2). 以 $\mathrm{Ph}_{3} \mathrm{PAuCl}$ 作为催化剂时, 反应产率仅 有 $33 \%$. 当以 $\mathrm{I}_{2}$ 或 $\mathrm{ICl}$ 作为亲电试剂时, 反应产率并没 有明显提升(表 3, Entries 4 6). 以 AgOTf 为催化剂, 在 在 1,2-二氯乙烷(DCE) 溶剂中加入 1.5 equiv. 的 NIS 为亲
电试剂时，反应以 $65 \%$ 的分离产率得到产物 $8 \mathbf{a}$ (表 3 , Entry 9).

表 3 条件篮选及优化

Table 3 Optimization of the reaction conditions

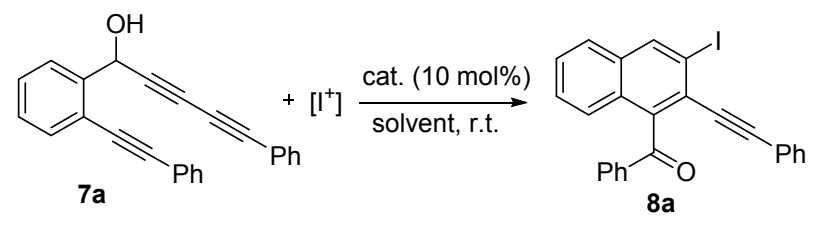

\begin{tabular}{|c|c|c|c|c|}
\hline Entry & Cat. & {$\left[\mathrm{I}^{+}\right]$(equiv.) } & Solvent & Yield $^{a} / \%$ \\
\hline 1 & $\mathrm{AgBF}_{4}$ & NIS (1.2) & DCM & 40 \\
\hline 2 & - & NIS (1.2) & DCM & $\mathrm{NR}^{b}$ \\
\hline 3 & AgOTf & NIS (1.2) & DCM & 50 \\
\hline 4 & $\mathrm{PPh}_{3} \mathrm{AuCl}$ & NIS (1.2) & DCM & 33 \\
\hline 5 & AgOTf & $\mathrm{I}_{2}(1.2)$ & DCM & 33 \\
\hline 6 & AgOTf & $\mathrm{ICl}(1.2)$ & DCM & 21 \\
\hline 7 & AgOTf & NIS (1.5) & DCM & 61 \\
\hline 8 & AgOTf & NIS (2.0) & DCM & 57 \\
\hline 9 & AgOTf & NIS (1.5) & DCE & 65 \\
\hline
\end{tabular}

随后，我们对底物的普适性进行了考察(表 4). 除 NIS 之外, $N$-溴代丁二酰亚胺(NBS)作为亲电试剂也能 很好地参与反应生成相应的目标产物. 此外, $\mathrm{R}^{1}$ 所在的 母体苯环对电子效应不是很敏感, 连接有吸电子或者推 电子基团的反应底物均能很好地与亲电试剂 NXS 发生 环化反应，并以中等到良好的产率得到目标化合物 $\mathbf{8 b} \sim \mathbf{8 e}($ 表 4, Entries 1 3). 研究还发现, 反应对于各类 不同电性的有机官能团兼容性良好. 例如，当 $\mathrm{R}^{2}$ 连接有 给电子基时，产率只能达到 $42 \%$, 而 $\mathrm{R}^{2}$ 连接如 $\mathrm{F} 、 \mathrm{Cl}$ 、 $\mathrm{COOMe}$ 以及张力比较大的环丙基均可得到兼容，以 $37 \% \sim 78 \%$ 的分离产率得到预期产物 $\mathbf{8 f} \sim \mathbf{8 k}$. 值得注意 的是，脂肪族基团如正丁基在反应中不受影响，能以 $38 \%$ 的产率分离得到预期产物 81 .

杂环化合物也可以在此反应中得到兼容. 当以吡啶 环取代的联三炔醇 9 作为反应物时，可以中等的产率得 到密集三取代的喹啉类衍生物 10 . 另外, 在此反应中还 分离到的主要副产物为 6,8-二碘代-7-炔基喹啉 $\mathbf{1 0}^{[23]}$ (表 5). 众所周知, 喹啉类化合物也是具有广泛生物或药 物活性的 “优势骨架(privileged structure)” 分子. 但是, 由于氮原子的钝化作用，喹啉环本身的反应性比较差， 在其骨架上同时引入反应性活泼的卤素、炔基和酮羰基 是非常困难的. 我们发展的这个串联亲电环化反应原料 易于制备，条件十分温和，因此，为喹啉类化合物的合 成提供了一种非常直接的思路.

如前所述，上述反应所得到的密集三取代的萗衍生 物 9 分子骨架上含有三种反应性良好的取代基：碘原 子、炔基和酮基. 组合这三种取代基可发生一些很有意 
表 4 底物拓展 ${ }^{a}$

Table 4 Substrate scope
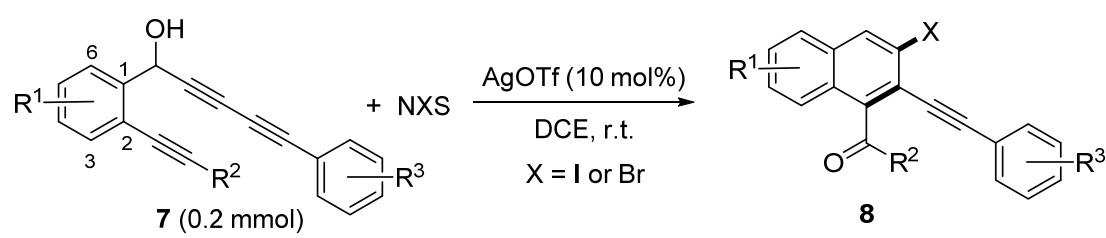

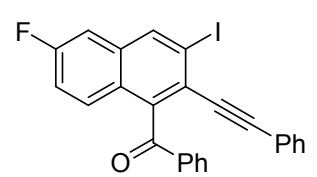

8b, $67 \%$

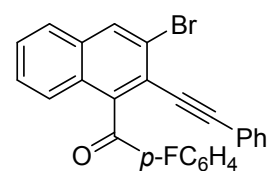

$8 g, 48 \%$<smiles>O=C(c1ccccc1)c1c(Br)cc2cc(F)ccc2c1-c1ccccc1</smiles>

$8 c, 60 \%$<smiles>O=C(OCc1ccccc1)c1c(C#Cc2ccccc2)c(I)cc2ccccc12</smiles>

8 h, $78 \%$<smiles>C#CC#Cc1c(I)cc2ccccc2c1C(C)=O</smiles>

8 e, $42 \%$<smiles>CC(=O)c1ccc(C(=O)c2c(C#Cc3ccccc3)c(I)cc3ccccc23)cc1</smiles>

8j, $58 \%$<smiles>CC#CC#Cc1c(I)cc2ccccc2c1C(=O)OCC</smiles>

8f, $67 \%$

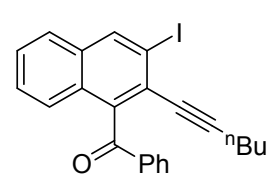

8I, $38 \%$<smiles>CCCCCCCCCCCC(=O)c1c(C#Cc2ccc(C)cc2)c(I)cc2ccccc12</smiles>

$8 \mathrm{~m}, 62 \%$<smiles>C=C(OC(=O)c1ccccc1)c1c(Br)cc2ccccc2c1-c1ccccc1</smiles>

8i, $60 \%$<smiles>Cc1ccc(C#Cc2c(Br)cc3ccccc3c2C(=O)c2ccccc2)cc1</smiles>

8 n, $55 \%$

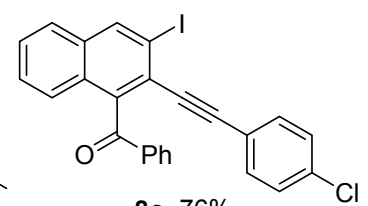

$80,76 \%$<smiles>O=C(c1c(C#Cc2ccccc2)c(I)cc2ccccc12)C1CC1</smiles>

$\mathbf{8 k}, 37 \%$

${ }^{a}$ Conditions: 7 (0.2 mmol), $\mathrm{X}^{+}$(0.3 mmol), AgOTf (10 mol\%), DCE (2 mL), $\mathrm{N}_{2}$, r.t.

表 5 基于吡啶的三炔醇底物的拓展

Table 5 Substrate scope that containing pyridine-based triynols

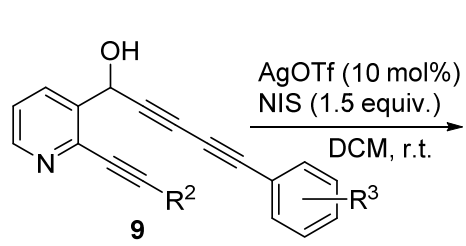

$R^{1}, R^{2}=$ aryl

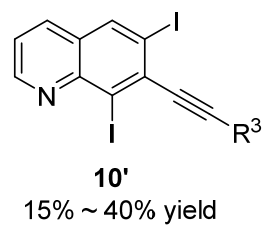

\begin{tabular}{|c|c|c|c|c|}
\hline Entry & $\mathrm{R}^{2}$ & $\mathrm{R}^{3}$ & Yield/\% (10) & Yield/\% $\left(\mathbf{1 0}^{\prime}\right)$ \\
\hline 1 & $\mathrm{Ph}$ & $\mathrm{Ph}$ & 45 (10a) & $38(\mathbf{1 0 a})$ \\
\hline 2 & 4- $\mathrm{FC}_{6} \mathrm{H}_{4}$ & $\mathrm{Ph}$ & 49 (10b) & $20(\mathbf{1 0 a})$ \\
\hline 3 & 4- $\mathrm{ClC}_{6} \mathrm{H}_{4}$ & $\mathrm{Ph}$ & $52(\mathbf{1 0 c})$ & $15(\mathbf{1 0 a})$ \\
\hline 4 & $\mathrm{Ph}$ & $4-\mathrm{FC}_{6} \mathrm{H}_{4}$ & 38 (10d) & 40 (10d') \\
\hline 5 & $\mathrm{Ph}$ & 4- $\mathrm{ClC}_{6} \mathrm{H}_{4}$ & $41(\mathbf{1 0 e})$ & $35\left(\mathbf{1 0} \mathbf{e}^{\prime}\right)$ \\
\hline
\end{tabular}

义的衍生反应，构建结构上更加复杂的多环芳香化合 物. 例如, 以化合物 9a 作为反应物, 在 $\operatorname{Pd}(\mathrm{OAc})_{2}$ 的催化 下可与苯胺发生反应得到苯并吲哚 $\mathbf{1 3}^{[24]}$. 化合物 9a 还 可以与对甲基苯乙炔在 $\mathrm{Cu}(\mathrm{OTf})_{2}$ 的催化下发生反应，生 成多取代菲衍生物 14 和 $\mathbf{1 6}^{[25]}$ (Scheme 3).

\section{3 铂催化的 1,6-二炔醇酯化合物的串联亲电环 异构化反应合成亚甲基狮酮衍生物}

狮骨架，包括亚甲基狮，长久以来即被视作 “优势 骨架”。含有狮核心结构的化合物广泛存在于各类天然 产物，或者具有重要生物或药物活性的分子当中，在农 药和材料科学领域也有大量的有机分子含有狮骨架结 构 $^{[26]}$. 因此，发展一些简单快捷的方法合成含狮结构的 化合物具有十分重要的意义.

事实上, 迄今为止人们已经发展出了多种方法合成 含狮结构的化合物. Scheme 4 中列举了一些有代表性的 通过分子间或分子内的串联反应合成亚甲基狮类化合 物的方法. 其中包括: 基于过渡金属如 $\mathrm{Au}^{[27 \mathrm{a} \sim 27 \mathrm{c}]}$, $\mathrm{Pd}^{[27 \mathrm{~d} \sim 27 \mathrm{f}]}, \mathrm{Rh}^{[27 \mathrm{~g}]}$ 等的催化体系构建亚甲基狮化合物的 方法，利用质子酸 ${ }^{[27 h]}$ 以及利用加热或光照的方式诱导 的自由基合成 ${ }^{[27 i, 27 j]}$ 等.

基于前述 NIS 可在过渡金属的活化下提供高度亲 电活性 $\mathrm{X}^{+}$的合成策略，具有亲电属性的酰基碳原子是 否也可以参与到上述 1,6-二炔醇的亲电环化反应中呢? 我们设想，若以酰基化的 1,6-二炔醇酯 17a 为反应底物, 该化合物在适当过渡金属 $[\mathrm{M}]$ 的催化下，可能发生分子 内的[3,3]- $\sigma$ 重排生成联烯酯中间体物种 $\mathbf{A}^{[28]}$. 该物种 $\mathbf{A}$ 

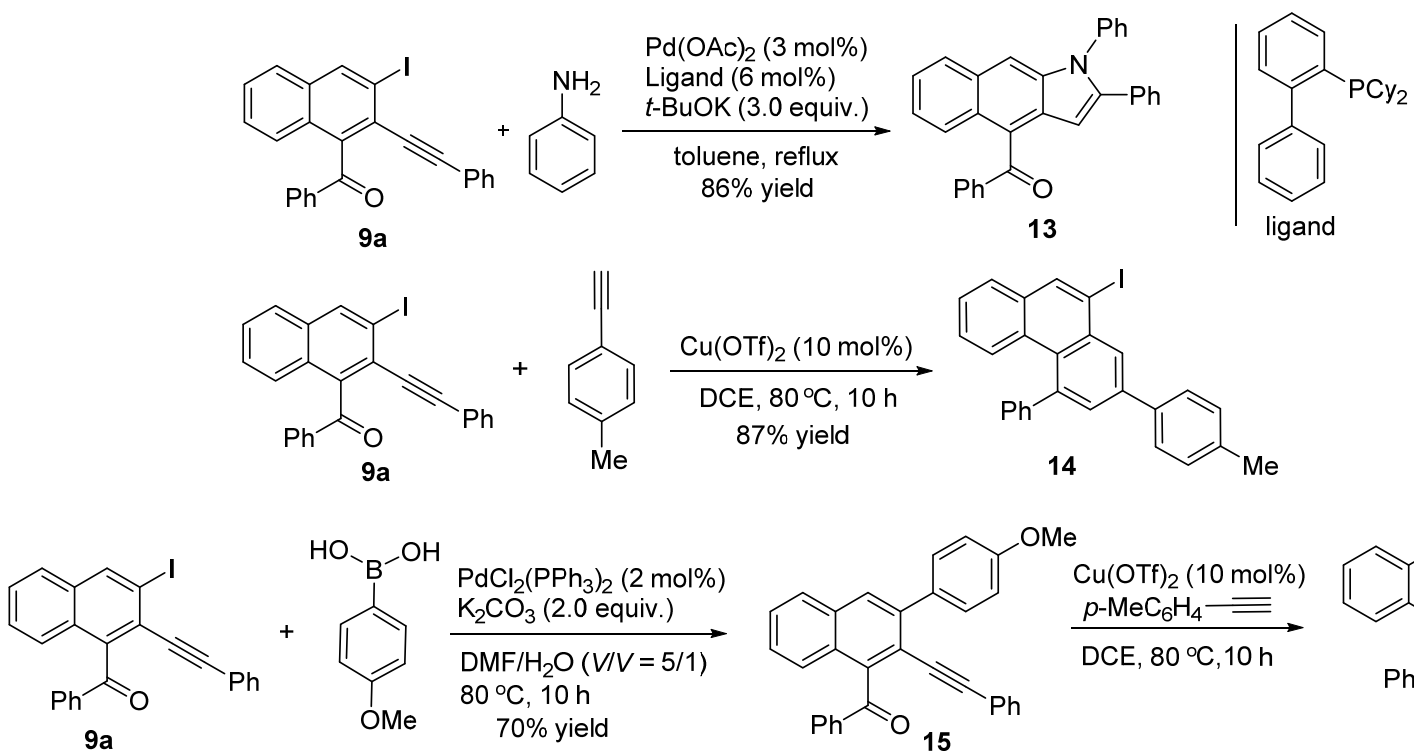

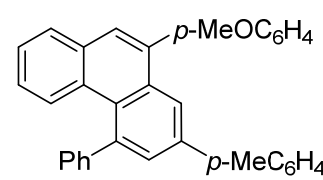

$16,94 \%$ yield

图式 3 密集三取代的萗衍生物 9a 的多样性转化反应

Scheme 3 Divergent transformations of the densely 1,2,3-trifunctionalized naphthalene 9a<smiles>[R]CC#Cc1ccccc1C#CCC</smiles><smiles>[R]OCC1(c2ccccc2)CC1c1ccccc1</smiles><smiles>[R]C#Cc1ccccc1C=C(C[R])C[R]</smiles>

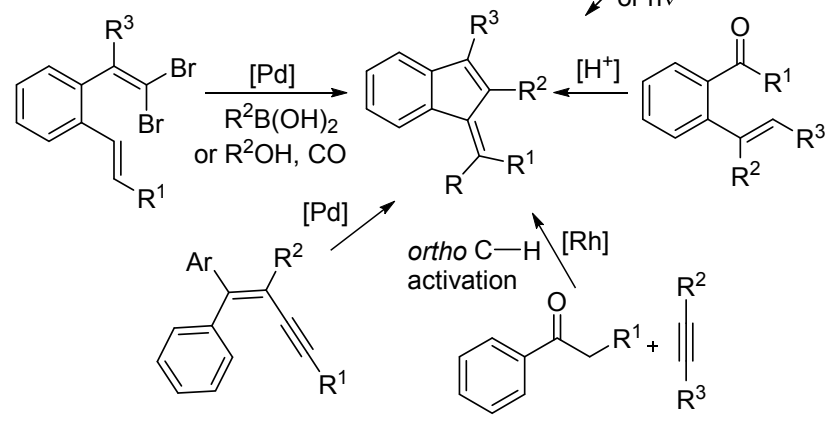

图式 4 一些合成亚甲基狮的传统方法

Scheme 4 Tradition methods for the synthesis of methylene indenes

中的金属催化剂 $[\mathrm{M}]$ 既可以与邻位的炔基配位又可以与 联烯键配位形成螯合络合物结构，即对反应底物的局部 加以活化. 在酯基氧原子的推动下, 联烯基可作为亲核 试剂对金属活化的炔基参键进行 5-exo-dig 顺式的进攻 得到中间体 $\mathbf{B}$, 最后可能经历分子内的超远程 1,5-酰基 迁移得到亚甲基茚酮类目标化合物 18a (Scheme 5) ${ }^{[11]}$.

以化合物 17a 为起始原料, 我们对该金属催化反应 的条件如催化剂、溶剂和添加剂进行了考察(表 6), 发现 当使用 $\mathrm{Au}$ 和 $\mathrm{Ag}$ 催化剂的时候没有得到预期的含狮结 构的产物. 令我们高兴的是, 当以 $\mathrm{PtCl}_{2}(10 \mathrm{~mol} \%)$ 为催 化剂, $80{ }^{\circ} \mathrm{C}$ 温度下反应, 可以 $33 \%$ 的产率得到预期产物 18a. 我们接着对 $[\mathrm{Pt}]$ 催化剂、溶剂和各种可能影响

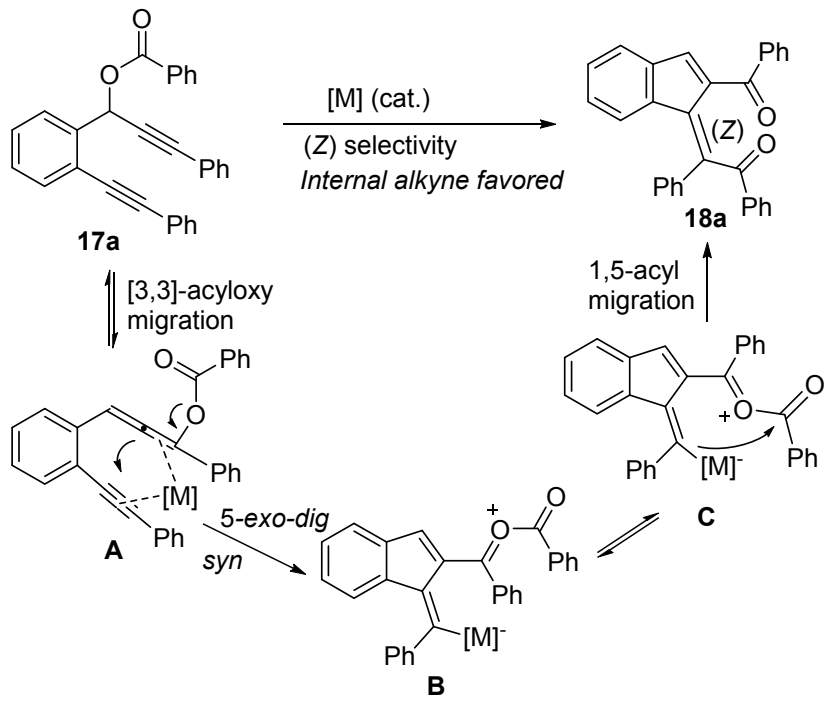

图式 5 合成亚甲基狮二酮的设想路线

Scheme 5 Proposed method for the synthesis of benzofulvene diketones

反应的添加剂进行一系列篮选，产率升高到了 $79 \%$ (表 6, Entries 1 10). 意外的是各种添加物对反应活性的影 响并不明显，例如，曾有大量的文献报道一氧化碳对于 铂类金属催化剂具有络合能力，能稳定活性铂物种，然 而在我们的反应中, 在 CO $(101 \mathrm{kPa})$ 的氛围里面产率并 没有明显提升 ${ }^{[29]}$; 此外, 实验还发现氧气以及各类配体 对反应产率的影响有限(表 6, Entries 11 13). 上述反应 只需要加入少量催化剂和溶剂即可顺利完成整个催化 转化，因此在工业上或许有潜在的合成价值.

接下来, 我们对底物的普适性进行了研究(表 7). 首 先，我们考察了反应底物 17 中，各种不同结构的 $\mathrm{R}^{1}$ 基 
表 6 条件筛选及优化 ${ }^{a}$

Table 6 Optimization of the reaction conditions<smiles>O=C(OC(C#Cc1ccccc1)c1ccccc1)c1ccccc1</smiles>
o-xylene, $80^{\circ} \mathrm{C}, 12 \mathrm{~h}$ 17a $\mathrm{Ph}$

$8 a$

\begin{tabular}{clclc}
\hline Entry & {$[\mathrm{Pt}](\mathrm{mol} \%)$} & Additive & Solvent & Yield $^{a} / \%$ \\
\hline 1 & $\mathrm{PtCl}_{2}(10)$ & - & DCE & 33 \\
2 & $\mathrm{PtCl}_{2}(10)$ & - & Toluene & 58 \\
3 & $\mathrm{PtCl}_{2}(10)$ & - & $\mathrm{PhCl}$ & 59 \\
4 & $\mathrm{PtCl}_{2}(10)$ & - & $o$-Xylene & 73 \\
5 & $\mathrm{PtCl}_{2}(5)$ & - & $o$-Xylene & 79 \\
6 & $\mathrm{PtCl}_{2}(2.5)$ & & $o$-Xylene & 65 \\
7 & $\left.\mathrm{Pt}^{\mathrm{acac}}\right)_{2}(5)$ & & $o$-Xylene & 49 \\
8 & $\mathrm{PtCl}_{4}(5)$ & & $o$-Xylene & 27 \\
9 & $\mathrm{PtPh}_{4}(5)$ & & $o$-Xylene & $\mathrm{Trace}$ \\
10 & $\mathrm{PtCl}_{2}(5)$ & $\mathrm{CO}$ & $o$-Xylene & 74 \\
11 & $\mathrm{PtCl}_{2}(5)$ & $\mathrm{O}$ & $o$-Xylene & 64 \\
12 & $\mathrm{PtCl}_{2}(5)$ & $\mathrm{dppp}(10$ mol\%) & $o$-Xylene & 53 \\
\hline
\end{tabular}

${ }^{a}$ 分离产率: 以 $17 \mathrm{a}$ 为基准.

团在超远程 1,5-酰基迁移过程中的影响： $\mathrm{R}^{1}$ 所在的芳香 环上的甲氧基(18b) 和硝基(18c)均能顺利发生迁移反应, 分别以 $83 \%$ 和 $81 \%$ 的产率得到对应结构的产物 $\mathbf{1 8 b}$ 和 18c. 当 $\mathrm{R}^{1}$ 是 $\beta$-荎基(18d)、苯乙烯基(18e)、环已基(18f)、 呋喃基 (18g) 和二甲氨基(18h) 时, 都有较理想的产率 (50\% 78\%). 对 $\mathrm{R}^{2}$ 基团考察发现，连接给电子基(18j) 和吸电子基(18i)的产率都可以达到 70\% 72\%. 最后对 $\mathrm{R}^{3}$ 和 $\mathrm{R}^{4}$ 基团考察, $\mathrm{R}^{3}$ 连接醚键结构的产物(18I)产率为 $76 \%, \mathrm{R}^{4}$ 是大位阻的叔丁基时, 对应预期产物 $18 \mathrm{~m}$ 的收 率也比较理想. 有趣的是, 当母体环是喹啉时, 在标准 条件下的目标产物 $18 \mathrm{n}$ 产率只有 $26 \%$, 不过当反应条件 改为在 CO (101 kPa)氛围下, 催化剂为 $10 \mathrm{~mol} \%$, 并且 温度升高到 $100{ }^{\circ} \mathrm{C}$ 时能顺利地发生转化, 以 $64 \%$ 的产 率得到目标产物 18n(表 7).

为了进一步验证反应的机理, 我们进行了官能团交 叉实验. 以等物质的量的 19 和 21 混合物作为反应底物, 在标准反应条件下一锅法反应, 分别以 $82 \%(\mathbf{2 0 a})$ 和 65\% (22a)的产率分离到相应目标产物. 酰基交叉迁移 的产物 20b 和 22b 并没有生成, 表明此反应所涉及的超 远程 1,5-酰基迁移历程为分子内迁移的历程, 迁移前后 并不涉及酰基碳碳键的断裂(Eq. 8).

另外, 为了验证两个羰基氧原子的的来源, 我们开 展了 ${ }^{18} \mathrm{O}$ 同位素试验, 反应产物经 ${ }^{13} \mathrm{C} \mathrm{NMR}, \mathrm{HMBC}$ 以 及 HRMS 检测证实, 反应所得到的产物中两个羰基氧 原子均来源于反应底物分子中的酯基(Eq. 9). 由此实验 事实, 最终确认了我们先前所设想的预期反应的机理
表 7 底物拓展 ${ }^{a}$

Table 7 Substrate scope
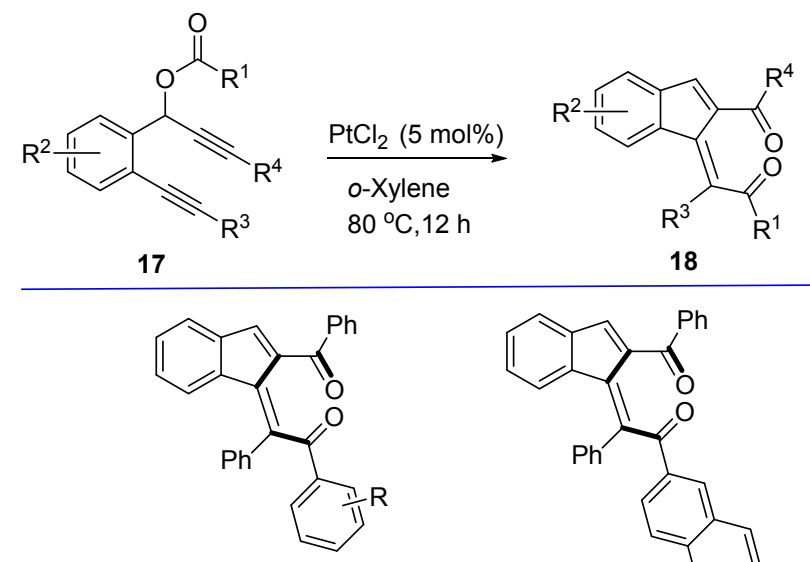

$$
\text { 18b }(\mathrm{R}=4-\mathrm{MeO}), 83 \%
$$$$
18 \mathrm{c}\left(\mathrm{R}=4-\mathrm{NO}_{2}\right), 81 \%
$$<smiles>CC(C)C(C)(C)C</smiles><smiles>CC(C)(C)OC(=O)C1=Cc2ccccc2C1=C(C(=O)C=Cc1ccccc1)c1ccccc1</smiles><smiles>O=C(C(=C1C(C(=O)C2CCCCC2)=Cc2ccccc21)c1ccccc1)c1ccccc1</smiles>

$18 f, 45 \%$<smiles>O=C(C(=C1C(C(=O)c2ccco2)=Cc2ccccc21)c1ccccc1)c1ccccc1</smiles><smiles>CN(C)C(=O)/C(=C1\C(C(=O)c2ccccc2)=Cc2ccccc21)c1ccccc1</smiles>

$18 \mathrm{~g}, 50 \%$<smiles></smiles>
18i, $72 \%$<smiles></smiles>

$18 k, 79 \%$<smiles>CC(C)(C)C(=O)C1=Cc2ccccc2C1=C(C(=O)c1ccccc1)c1ccccc1</smiles><smiles>O=C(C(C(=O)c1ccccc1)=C1C=Cc2cc3ccccc3nc21)c1ccccc1</smiles>

$18 \mathrm{~m}, 77 \%$

$18 n, 64 \%^{b}$ 


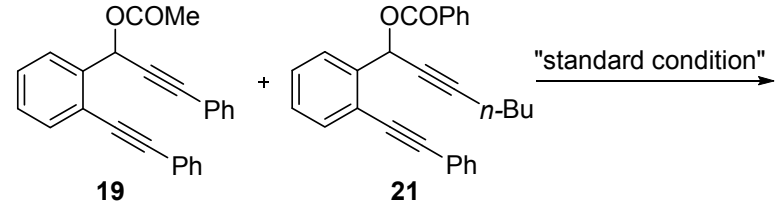<smiles>CC(=O)/C(=C1\C(C(=O)c2ccccc2)=Cc2ccccc21)c1ccccc1</smiles>

20a, $82 \%$

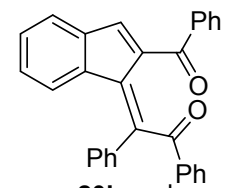
20b, n.d. 22a, $65 \%$

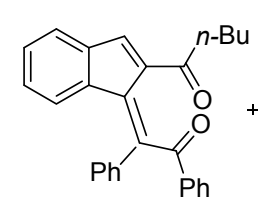<smiles>CCCCC(=O)C1=Cc2ccccc2C1=C(C(C)=O)c1ccccc1</smiles>

22b, n.d.
(Scheme 5).

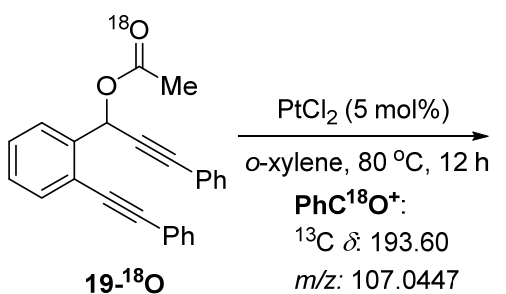<smiles>CC(=O)/C(=C1/c2cc3ccccc3cc2C(=O)OC1c1ccccc1)c1ccccc1</smiles>

$20 \mathrm{a}-{ }^{18} \mathrm{O}$
最近, 我们小组的研究还发现, 当以非环化的 1,5二炔醇酯 23a 作为反应底物时, 在 $\mathrm{PtCl}_{2}$ 的催化条件下 可得到狮 2-位烯基化的亚甲基苑酯衍生物 24a (Eq. 10). 产物的结构经过单晶衍射实验进行了证实 ${ }^{[30]}$. 我们推 测反应的机理可能首先涉及到 $[\mathrm{Pt}]$ 催化的分子内的 $[3,3]-\sigma$ 重排得到一个联烯酯中间体物种, 随后发生分子 内的环异构化以及随之发生的 1,5 -氢迁移反应而得到最 终产物. 相关的实验探索以及机理验证研究正在进行当 中 $^{[31]}$.<smiles>CC(=O)OC(C)(C)C#Cc1ccccc1C#Cc1ccccc1</smiles>

23a

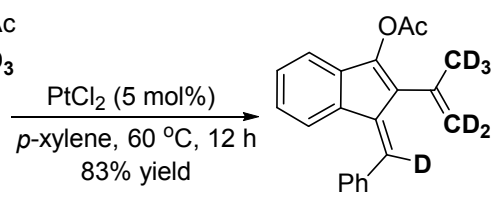

24a

\section{4 三氯化铋促进的 1,5-二炔类化合物的串联亲 电环化反应合成三氟甲硫基亚甲基狮衍生物}

含氟的化合物往往具有优越的生物和药物活性, 是 先导药物研发中公认的 “明星分子”. 而三氟甲硫基 $\left(\mathrm{CF}_{3} \mathrm{~S}\right)$ 取代的有机化合物由于具有非常高的亲油能力 (lipophilicity, $\pi_{\mathrm{R}}=1.44$ ) 以及强电负性 ${ }^{[32]}$, 引起了药物化 学家和有机合成化学家的注意. 对于含氟化合物的合成
方法学研究目前也是国际有机化学研究的热点领域之 一. 例如, 目前已经成功证实的含有三氟甲硫基结构片 段的药物有: Tiflorex, Fipronil 以及 Triflumidate 等 ${ }^{[33]}$ (图 2).

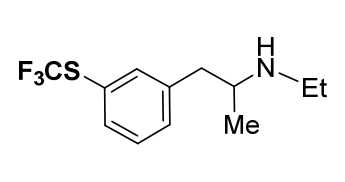

Tiflorex<smiles>N#Cc1nn(-c2c(Cl)cc(C(F)(F)F)cc2Cl)c(N)c1S(=O)(=O)C(F)(F)F</smiles>

Fipronil<smiles>CCOC(=O)N(c1cccc(C(=O)c2ccccc2)c1)S(=O)(=O)C(F)(F)F</smiles>

图 2 一些含三氟甲硫基结构的药物分子

Figure 2 Some marketed drugs that containing $\mathrm{CF}_{3} \mathrm{~S}$ moieties

早期，合成含三氟甲硫基官能团有机化合物的方式 是采用亲核性的金属试剂, 如 $\mathrm{CF}_{3} \mathrm{~S}-\mathrm{M}(\mathrm{M}=\mathrm{Cu}, \mathrm{Ag}$, TMS, $\mathrm{NMe}_{4}$ )作为反应试剂 ${ }^{[34]}$, 使之在过渡金属催化的 条件下发生类似转金属化反应而引入. 这种亲核环化的 方法合成效率比较高, 反应选择性好, 不过缺点是需要 对反应底物进行 “预官能团化”，这样无疑需要增加额 外的合成操作; 并且很多时候由于金属试剂 $\mathrm{CF}_{3} \mathrm{~S}-\mathrm{M}$ 的 存在，一些敏感基团如羰基无法兼容. 另外一种新型的 采用亲电性氟试剂的合成方法是, 利用三氟甲硫胺作为 反应物，该物质在反应条件下 “原位” 生成高度亲电性 的三氟甲硫基正离子 $\left(\mathrm{CF}_{3} \mathrm{~S}^{+}\right)$, 从而诱导亲电环化反应, 合成三氟甲硫化的有机化合物 ${ }^{[35]}$.

最近的研究发现在 $\mathrm{BiCl}_{3}$ 的催化下, 邻炔基苯磺酰 胺可与三氟甲硫基苯胺发生反应，高效得到三氟甲硫化 的苯并噻嗪衍生物 ${ }^{[36]}$. 我们设想高度亲电活性的三氟 甲硫基正离子 $\left(\mathrm{CF}_{3} \mathrm{~S}^{+}\right)$是否也能参与到非环化的 $1, n$-二 炔基化合物 25 的串联亲电环化反应中来. 原位生成的 $\mathrm{CF}_{3} \mathrm{~S}^{+}$可首先与底物 $\mathbf{2 5}$ 发生亲电环化反应, 得到碳正离 子中间体，高反应活性的碳正离子中间体可迅速被反应 体系内的亲核试剂 $\mathrm{Nu}$ 捕获, 最终得到三氟甲硫化的亚 甲基狮衍生物 26 (Scheme 6). 此方法的成功实施, 有利 于我们成功发展一例合成 3-三氟甲硫基亚甲基茆衍生 物的方法，反应具有十分高的效率以及良好的化学和区 域选择性 ${ }^{[12]}$.

我们以化合物 $25 \mathrm{a}$ 和三氟甲硫基苯胺作为起始反应 物, 对反应条件进行了篮选, 结果列于表 8. 在 1,2-二氯 乙烷(DCE)作为反应溶剂时, 当加入 2.0 equiv. $\mathrm{FeCl}_{3}$ 时, 我们以 $40 \%$ 的分离产率分离到了一个含氯原子的产物 26a (表 8, Entry 1). 产物中的氯元素可能来自于铁盐. 当加入 6.0 equiv. $\mathrm{NaCl}$ 取代 $\mathrm{FeCl}_{3}$ 时, 没有任何反应发 


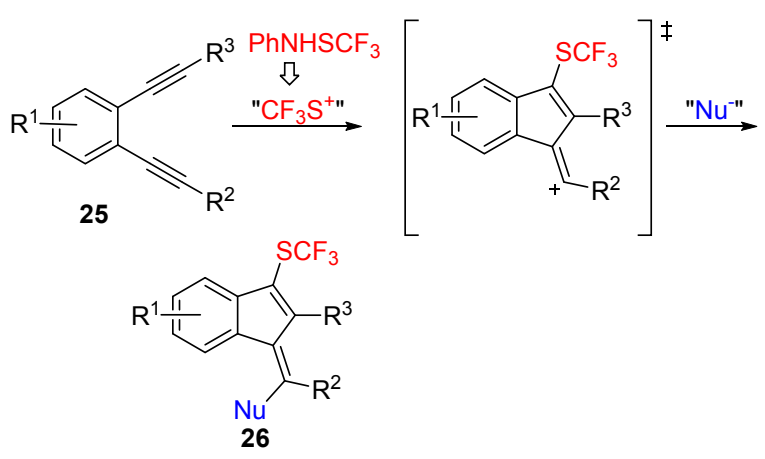

图式 6 反应设计

Scheme 6 Research design

生, 此结果表明金属阳离子的存在对反应起到了关键的 作用(表 8, Entry 2). 随后, 我们篮选了各类不同的 Lewis 酸催化剂, 实验发现当加入 3.0 equiv. $\mathrm{BiCl}_{3}$ 时, 能 以几乎定量的产率得到 26a(表 8, Entries 3 6). 考虑到 金属铋盐价格比较昂贵, 我们随后尝试了加入 1.0 equiv. $\mathrm{BiCl}_{3}$, 并添加 8.0 equiv. $\mathrm{Cl}^{-}$到反应体系, 结果发现反应 效率均降低明显(表 8, Entries 7 9). 当不加路易斯酸催 化剂时, 没有任何反应发生(表 8, Entry 10).

表 8 条件篮选及优化 ${ }^{a}$

Table 8 Optimization of the reaction conditions<smiles>CN(/C(=C1\C(c2ccccc2)=C(C(C)(C)C)c2ccccc21)c1ccccc1)c1ccccc1</smiles>

26a 26a' (not observed)

\begin{tabular}{|c|c|c|}
\hline Entry & $\mathrm{MCl}_{n}$ & Yield $^{a} \%$ \\
\hline 1 & $\mathrm{FeCl}_{3}(2.0$ equiv. $)$ & 40 \\
\hline 2 & $\mathrm{NaCl}$ (6.0 equiv.) & N.R. \\
\hline 3 & $\mathrm{InCl}_{3}$ (2.0 equiv.) & 20 \\
\hline 4 & $\mathrm{BiCl}_{3}(2.0$ equiv. $)$ & 66 \\
\hline 5 & $\mathrm{BiCl}_{3}$ (1.0 equiv.) & 41 \\
\hline 6 & $\mathrm{BiCl}_{3}$ (3.0 equiv.) & 98 \\
\hline 7 & $\mathrm{BiCl}_{3}$ (1.0 equiv. $) / \mathrm{LiCl}$ (8.0 equiv.) & 31 \\
\hline 8 & $\mathrm{BiCl}_{3}$ (1.0 equiv. $) / \mathrm{MgCl}_{2}$ (4.0 equiv.) & 57 \\
\hline 9 & $\mathrm{BiCl}_{3}$ (1.0 equiv. $) / \mathrm{CaCl}_{2}$ (4.0 equiv.) & 36 \\
\hline 10 & $\mathrm{BiCl}_{3}(1.0$ equiv. $) / n-\mathrm{Bu}_{4} \mathrm{NCl}$ (4.0 equiv.) & 0 \\
\hline 11 & - & N.R. \\
\hline
\end{tabular}

此反应并没有分离到苯胺作为亲核试剂捕获亚甲 基茆正离子的产物 26a'. 这可能是由于反应过程中, 三 氟甲硫基苯胺分子内亲核性的氮原子首先进攻 Lewis 酸 性的 $\mathrm{Bi}^{3+}$, 随后所得到的两性离子中间体 $\mathbf{A}$ 发生分子内
的解离, 分别生成亲电试剂 $\left(\mathrm{CF}_{3} \mathrm{~S}^{+}\right)$、亲核试剂 $\mathrm{Cl}^{-}$, 以 及苯胺基二氯化铋物种 B (Scheme 7).

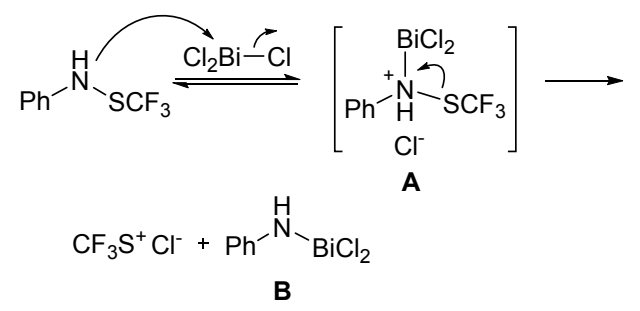

图式 7 可能的反应机理

Scheme 7 Proposed mechanism

在得到了优化的反应条件之后, 我们对此反应进行 了底物拓展. 各类卤素都可以很好的参与反应，化合物 26b $\sim 26 d$ 的产率中等到优秀. 另外，推电子的二甲基以 及拉电子的酯基均可以很好的参与反应，以 $50 \% \sim 80 \%$ 的产率生成相应目标产物. 含有噻吩杂环的氯化亚甲基 三氟甲硫狮 26g 也可以可接受的产率合成出来(表 9).

表 $9 \mathrm{BiCl}_{3}$ 促进的亲电环化反应合成三氟甲硫基狮 ${ }^{a}$ Table $9 \mathrm{BiCl}_{3}$-mediated electrophilic cyclization for the generation of trifluoromethanesulfanyl benzofulvenes<smiles>[R]C#Cc1ccccc1C#C[R]</smiles>

26b, $99 \%$

26c, $91 \%$<smiles>FC(Br)=C(Cl)C1=C(C=CBr)C(=C(Br)Br)c2ccccc21</smiles>

26d, $63 \%$<smiles></smiles>

26e, $80 \%$<smiles>COCCC1=C(C=CC(=O)OCc2ccccc2)c2ccccc2C1=C(Cl)C(F)(F)F</smiles><smiles>FC(F)(F)C1=C(c2cccs2)C(=C(Cl)c2cccs2)c2ccccc21</smiles>

26f, $53 \%$

26g, $51 \%$

${ }^{a}$ Conditions: 25 (0.2 mmol), $\mathrm{PhNHSCF}_{3}(0.4 \mathrm{mmol}), \mathrm{BiCl}_{3}(0.6 \mathrm{mmol}), \mathrm{DCE}$ $(2 \mathrm{~mL}), \mathrm{N}_{2}, 80{ }^{\circ} \mathrm{C}, 16 \mathrm{~h}$.

我们还成功拓展了此反应的合成范围. 利用对甲苯 磺酸 $\mathrm{TsOH}$ 与 $1,5-$ 二炔以及三氟甲硫基苯胺的无金属三 组分反应，可以一锅法高选择性合成对甲苯磺酰氧基取 代的三氟甲硫基狮衍生物 27. 反应中，对甲苯磺酸 
$(p-\mathrm{TsOH})$ 可提供亲核性的甲苯磺酰氧基 $\left(\mathrm{TsO}^{-}\right)$, 此负离 子可作为亲核试剂参与到三氟甲硫基正离子诱导的亲 电环化反应当中 ${ }^{[37]}$. 底物适用性试验结果表明, 反应对 各类推电子或拉电子的官能团兼容性良好，能以 $30 \%$ 90\%的产率生成各类 3-三氟甲硫基亚甲基荫化合物 27(表 10).

表 $10 \mathrm{TsOH}$ 促进的亲电环化反应合成三氟甲硫基苑 Table 10 TsOH-promoted electrophilic cyclization for the generation of trifluoromethanesulfanyl benzofulvenes

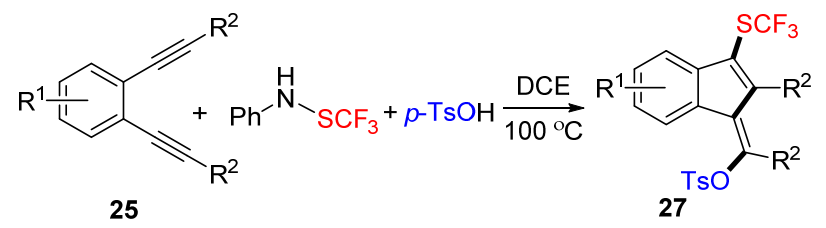

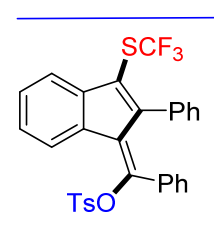

27a, $90 \%$

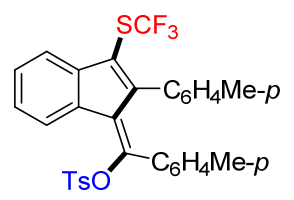

27d, $30 \%$

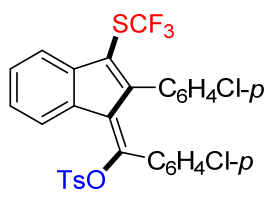

27b, $80 \%$

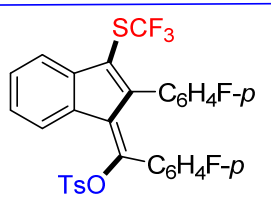

27c, $75 \%$

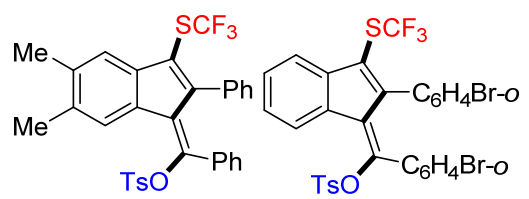

27f, $56 \%$

${ }^{a}$ Conditions: $25(0.2 \mathrm{mmol}), \mathrm{PhNHSCF}_{3}(0.4 \mathrm{mmol}), p$-TsOH $(0.6 \mathrm{mmol})$, DCE $(2 \mathrm{~mL}), \mathrm{N}_{2}, 100{ }^{\circ} \mathrm{C}, 12 \mathrm{~h}$.

值得注意的是, 产物 26 和 27 中所附带的各类卤素 原子以及对甲苯磺酰氧基 $\left(\mathrm{TsO}^{-}\right)$, 均能够作为潜在的合 成子进行进一步的官能团化, 从而引入各类其他的官能 团.

\section{5 结论与展望}

非环状的多炔基化合物是一类具有高度不饱和性 的有机化合物, 这类化合物反应活性高, 反应位点多. 在过渡金属催化的作用下，该类化合物可与各类亲电试 剂发生高选择性串联环异构化反应, 合成不同结构的芳 香化合物. 利用过渡金属催化具有 1,6-二炔醇或 1,3,5三炔醇类化合物与亲电试剂(NXS)的串联环化反应, 我 们发展了一类在温和的反应条件下, 高化学选择性地构 建卤代苯并 $[a]$ 芴醇和密集三取代的卤代炔基萗酮衍生 物的合成方法. 通过对底物的简单调控, 发现 1,6-二炔 酯化合物中羰基官能团可作为亲电试剂诱导分子内的 串联环异构化反应，合成具有新型结构的亚甲基茚二酮 类以及 2-位烯基化的亚甲基苑衍生物. 结合三氟甲硫基 苯胺可原位生成三氟甲硫基正离子及其所具有的高亲 电活性特性, 我们发展了 $\mathrm{BiCl}_{3}$ 或 $p-\mathrm{TsOH}$ 促进的 1,5-二
炔类化合物与三氟甲硫基苯胺的串联环化反应，高化学 和区域选择性地构建了具有三氟甲硫基亚甲基狮骨架 结构衍生物.

尽管对上述反应的使用范围以及拓展进行了一些 初步探索，但是在完善和提高上述反应的合成效率，以 及提高反应的不对称选择性方面仍有待于进一步研究. 此外，如何进一步拓展具有更多反应柔性的脂肪链状的 多炔基化合物与亲电试剂的环异构化反应，特别是在无 金属催化的二炔或三炔化合物的分子内官能团化反应 方面仍然需要加以探索. 搞清楚这些问题将有助于加深 对于反应机制的深入理解，并发展出具有新型环状结构 的有机化合物的合成方法.

\section{作者简介}

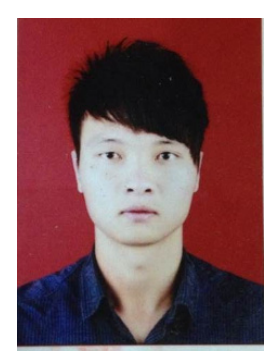

朱辉, 江西省抚州市人. 2013 年本科毕业于南昌大 学科学技术学院, 同年进入江西师范大学化学化工学院 攻读有机化学专业硕士学位. 目前主要从事过渡金属催 化的串联亲电环化反应构建类天然产物骨架方面的研 究.

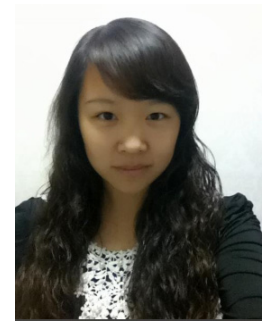

严冰玉, 河南省郑州市人. 2012 年 9 月进入江西师 范大学化学化工学院应用化学专业学习. 目前在陈知远 副教授课题组从事有机化学科研训练.

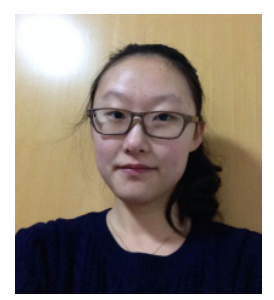

曹杨, 1994 年 3 月出生于江苏南通. 2012 年 9 月进 入江西师范大学化学化工学院应用化学专业学习. 目前 
在陈知远副教授课题组从事有机化学科研训练.

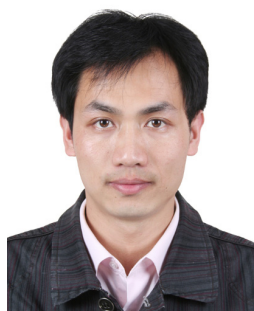

陈知远, 副教授. 2003 年本科毕业于华中农业大学 理学院, 2003 年至 2006 年在武汉大学化学与分子科学 学院学习, 获有机化学专业硕士学位. 2008 年进入复旦 大学化学系有机化学专业学习, 2011 年 6 月获理学博士 学位. 2011 年 7 月至今在江西师范大学化学化工学院任 教, 期间, 2012 年 8 月至 2013 年 8 月在新加坡南洋理工 大学(NTU) Naohiko Yoshikai 教授课题组做博士后研究. 目前的研究兴趣集中于基于 “绿色化学” 理念的碳杂环 有机小分子化合物的高选择性合成方面.

\section{References and notes}

[1] Michelet, V.; Toullec, P. Y.; Genet, J. Angew. Chem., Int. Ed. 2008, 47, 4268 .

[2] Denmark, S. E.; Thorarensen, A. Chem. Rev. 1996, 96, 137.

[3] Luo, Y.; Pan, X.; Yu, X.; Wu, J. Chem. Soc. Rev. 2014, 43, 836.

[4] (a) Schmidbaur, H.; Schier, A. In Science of Synthesis, Vol. 3, Ed.: Olneil, I. A., Thieme, Stuttgart, 2004, p. 691.

(b) Gimeno, M. C.; Laguna, A. In Comprehensive Coordination Chemistry II, Vol. 5, Eds.: Constable, E. C.; Dilworth, J. R., Elsevier, Oxford, 2004, p. 911.

(c) Díaz-Requejo, M. M.; Pérez, P. J. Chem. Rev. 2008, 108, 3379.

(d) Patil, N. T.; Yamamoto, Y. Chem. Rev. 2008, 108, 3395.

[5] (a) Fürstner, A. Chem. Soc. Rev. 2009, 38, 3208.

(b) Sohel, S. M.; Liu, R.-S. Chem. Soc. Rev. 2009, 38, 2269.

[6] Godoi, B.; Schumacher, R.; Zeni, G. Chem. Rev. 2011, 111, 2937.

[7] (a) Chen, Z.; Ye, C.; Zhu, H.; Zeng, X.; Yuan, J. Chem.-Eur. J. 2014, 20, 4237

(b) Ye, C.; Zhu, H.; Chen, Z.; Wu, J. J. Org. Chem. 2014, 79, 8900. (c) Li, Y.; Gao, L.; Zhu, H.; Li, G.; Chen, Z. Org. Biomol. Chem. 2014, 12, 6982 .

(d) Huang, P.; Chen, Z.; Yang, Q.; Peng, Y. Org. Lett. 2012, 14, 2790 .

(e) Huang, P.; Yang, Q.; Chen, Z.; Ding, Q.; Xu, J.; Peng, Y. J. Org. Chem. 2012, 77, 8092

[8] (a) Chen, Z.; Ding, Q.; Yu, X.; Wu, J. Adv. Synth. Catal. 2009, 351, 1692.

(b) Chen, Z.; Yu, X.; Su, M.; Wu, J. Adv. Synth. Catal. 2009, 351, 2702 .

[9] (a) Chen, Z.; Zeng, M.; Yuan, J.; Yang, Q.; Peng, Y. Org. Lett. 2012, 14, 3588 .

(b) Chen, Z.; Zeng, M.; Yang, Q.; Peng, Y. CN 102659512, 2012 [Chem. Abstr. 2012, 157, 492411].

[10] Chen, Z.; Jia, X.; Ye, C.; Qiu, G. Y.; Wu, J. Chem.-Asian J. 2014, 9, 126.

[11] Chen, Z.; Jia, X.; Huang, J.; Yuan, J. J. Org. Chem. 2014, 79, 10674.

[12] Xiao, Q.; Zhu, H.; Li, G.; Chen, Z. Adv. Synth. Catal. 2014, 356, 3809 .
[13] (a) Gould, S. J.; Tamayo, N.; Melville, C. R.; Cone, M. C. J. Am. Chem. Soc. 1994, 116, 2207.

(b) Mithani, S.; Weeratunga, G.; Taylor, N. J.; Dmitrienko, G. I. J. Am. Chem. Soc. 1994, 116, 2209.

[14] Gould, S. J.; Melville, C. R. Bioorg. Med. Chem. Lett. 1995, 6, 51.

[15] Gould, S. J.; Melville, C. R.; Cone, M. C.; Chen, J.; Carney, J. R. J. Org. Chem. 1997, 62, 320

[16] (a) Rodríguez, D.; Castedo, L.; Domínguez, D.; Saá, C. Tetrahedron Lett. 1999, 40, 7701

(b) Rodríguez, D.; Navarro, A.; Castedo, L.; Domínguez, D.; Saá, C. Org. Lett. 2000, $2,1497$.

(c) Rodríguez, D.; Navarro-Vázquez, A.; Castedo, L.; Domínguez, D.; Saá, C. Tetrahedron Lett. 2002, 43, 2717.

(d) Rodríguez, D.; Quintás, D.; García, A.; Saá, C.; Domínguez, D. Tetrahedron Lett. 2004, 45, 4711 .

[17] (a) Liu, L.; Zhang, J.-L. Angew. Chem., Int. Ed. 2009, 48, 6093.

(b) Liu, L.; Wei, L.; Zhang, J.-L. Adv. Synth. Catal. 2010, 352, 1920 .

[18] (a) Jin, T.; Yamamoto, Y. Org. Lett. 2007, 9, 5259

(b) Shi, M.; Horn, M.; Kobashiya, S.; Mayr, H. Chem.-Eur. J. 2009, 15,8533 .

[19] (a) Kitamura, T.; Kobayashi, S.; Taniguchi, H.; Rappoport, Z. J. Org. Chem. 1982, 47, 5003.

(b) Chen, X.; Lu, P.; Wang, Y.-G. Chem.-Eur. J. 2011, 17, 8105.

[20] (a) Meyers, A. I.; Willemsen, J. J. Chem. Commun. 1997, 1573.

(b) Kometani, T.; Takeuchi, Y.; Yoshii, E. J. Org. Chem. 1983, 48, 2630 .

(c) Lester, W. Annu. Rev. Microbiol.1972, 26, 85.

(d) Rinehart, K. L.; Jr. Acc. Chem. Res. 1972, 5, 57.

(e) Laatsch, H. Angew. Chem., Int. Ed. 1994, 106, 438.

[21] Fu, G.; Yoda, T.; Kasatani, K.; Okamoto, H.; Takenaka, S. Jpn. J. Appl. Phys. 2005, 44, 3945.

[22] Niu, R.-M.; Zhu, X.-R.; Sun, Z.-R. Acta Opt. Sinica 2003, 23, 18 (in Chinese). (牛瑞民, 朱小蓉, 孙真荣, 光学学报, 2003, 23, 18.)

[23] Wang, L.; Zhu, H.; Lu, L.; Yang, F.; Liu, X.-Y; Liang, Y.-M. Org Lett. 2012, 14, 1990.

[24] (a) Lavery, C. B.; McDonald, R.; Stradiotto, M. Chem. Commun. 2012, 48, 7277.

(b) Tang, Z.; Hu, Q.-S. Adv. Synth. Catal. 2006, 348, 846.

(c) Asao, N.; Nogami, T.; Lee, S.; Yamamoto, Y. J. Am. Chem. Soc. 2003, 125, 10921 .

[25] Asao, N. Synlett 2006, 1645.

[26] (a) Wang, Z.-G.; Chen, L. -Q.; Chen, J.-B.; Zheng, J. F.; Gao, W.-W.; Zeng, Z.-P.; Zhou, H.; Zhang, X.-K.; Huang, P.-Q.; Su, Y. Eur. J. Med. Chem. 2013, 62, 632.

(b) Karaguni, I. M.; Glusenkamp, K. H.; Langerak, A.; Geisen, C.; Ullrich, V.; Winde, G.; Moroy, T.; Muller, O. Bioorg. Med. Chem. Lett. 2002, 12, 709.

(c) Clegg, N. J.; Paruthiyil, S.; Leitman, D. C.; Scanlan, T. S. J. Med. Chem. 2005, 48, 5989 .

(d) Halder, S.; Satyam, A. Tetrahedron Lett. 2011, 52, 1179.

(e) Gao, H.; Katzenellenbogen, J. A.; Garg, R.; Hansch, C. Chem. Rev. 1999, 99, 723.

[27] (a) Ye, L.; Wang, Y.; Aue, D.; Zhang, L. J. Am. Chem. Soc. 2012, 134,31

(b) Hashmi, A.; Braun, I.; Nosel, P.; Schadlich, J.; Wieteck, M.; Rudolph, M.; Rominger, F. Angew. Chem. Int. Ed. 2012, 51, 4456.

(c) Li, C.; Zeng, Y.; Wang, J. Tetrahedron Lett. 2009, 50, 2956.

(d) Ye, S.; Gao, K.; Zhou, H.; Yang, X.; Wu, J. Chem. Commun. 2009, 5406.

(e) Ye, S.; Wu, J. Org. Lett. 2011, 13, 5980. 
(f) Hwang, J. H.; Jung, Y. H.; Hong, Y. Y.; Jeon, S. L.; Jeong, I. H. J. Fluorine Chem. 2011, 132, 1227.

(g) Patureau, F. W.; Besset, T.; Kuhl, N.; Glorius, F. J. Am. Chem. Soc. 2011, 133, 2154.

(h) Lee, P. S.; Fujita, T.; Yoshikai, N. J. Am. Chem. Soc. 2011, 133, 17283.

(i) Schmittel, M.; Vavilala, C. J. Org. Chem. 2005, 70, 4865.

(j) Bucher, G.; Mahajan, A. A.; Schmittel, M. J. Org. Chem. 2008, 73,8815 .

[28] (a) Zhang, G.; Catalano, V. J.; Zhang, L. J. Am. Chem. Soc. 2007, $129,11358$.

(b) Cho, E. J. Chem. Eur. J. 2012, 18, 4495.

[29] (a) Fürstner, A.; Davies, P. W.; Gress, T. J. Am. Chem. Soc. 2005, 127,8244 .

(b) Fürstner, A.; Davies, P. W. J. Am. Chem. Soc. 2005, 127, 15024.

(c) Fürstner, A.; Assa, C. J. Am. Chem. Soc. 2006, 128, 6306.

(d) Hsu, Y.; Ting, C.; Liu, R.-S. J. Am. Chem. Soc. 2009, 131, 2090.

(e) Lu, L.; Liu, X.; Shu, X.; Yang, K.; Ji, K.; Liang, Y. J. Org. Chem. 2009, 74, 474.

[30] CCDC1040967 (24a) contain the supplementary crystallographic data for this paper. These data can be obtained free of charge from The Cambridge Crystallographic Data Centre via www.ccdc.cam. ac.uk/data_request/cif.

[31] Unpublished results, manuscript under preparation.

[32] (a) Leo, A.; Hansch, C.; Elkins, D. Chem. Rev. 1971, 71, 525.

(b) Hansch, C.; Leo, A.; Taft, R. W. Chem. Rev. 1991, 91, 165.

[33] (a) Curtis-Prior, P.; Prouteau, M. Int. J. Obes. 1983, 7, 575. (b) Colliot, F.; Kukorowski, K.; Hawkins, D.; Roberts, D. Brighton Crop Prot. Conf. 1992, 1, 29.

(c) Hamon, N.; Shaw, R.; Yang, H. Proc.-Beltwide Cotton Conf. 1996, 2, 759 .

(d) Swingle, K. F.; Harrington, J. K.; Hamilton, R.; Kvam, D. C. Arch. Int. Phamacodyn. Ther. 1971, 192, 16.

[34] (a) Chen, C.; Xie, Y.; Chu, L.; Wang, R.; Zhang, X.; Qing, F.-L. Angew. Chem., Int. Ed. 2012, 51, 2492.

(b) Tyrra, W.; Naumann, D.; Hoge, B.; Yaguploskii, Y. L. J. Fluorine Chem. 2003, 119, 101.

(c) Man, E. H.; Coffmann, D. D.; Muetterties, E. L. J. Am. Chem. Soc. 1959, 81, 3575 .

(d) Adams, D. J.; Clark, J. H. J. Org. Chem. 2000, 65, 1456.

(e) Teverovskiy, G.; Surry, D. S.; Buchwald, S. L. Angew. Chem., Int. Ed. 2011, 50, 7312.

[35] (a) Wang, X.; Yang, T.; Cheng, X.; Shen, Q. Angew. Chem., Int. Ed. 2013, 52, 12860.

(b) Yang, Y.; Jiang, X.; Qing, F.-L. J. Org. Chem. 2012, 77, 7538.

(c) Bootwicha, T.; Liu, X.; Pluta, R.; Atodiresei, I.; Rueping, M. Angew. Chem., Int. Ed. 2013, 52, 12856.

(d) Ferry, A.; Billard, T. B.; Langlois, R.; Bacqué, E. Angew. Chem. Int. Ed. 2009, 48, 8551.

[36] Xiao, Q.; Sheng, J.; Chen, Z.; Wu, J. Chem. Commun. 2013, 49, 8647.

[37] Ferry, A.; Billard, T.; Langlois, R.; Bacqué. E. Angew. Chem., Int. Ed. 2009, 48, 8551. 